\title{
Collaborative learning in the COVID-19 pandemic: A change to the delivery of undergraduate nursing education
}

Patricia Thomson ${ }^{1,2}$, RN, MEd, CTLT, Senior Academic Staff Member

Anna Richardson ${ }^{1}$, RN, MPH (Dist.) DipTertTch, Principal Academic Staff Member

Gail Foster ${ }^{1}$, RN, MClinEd, PGDip Prof. Nursing Practice, Senior Academic Staff Member

${ }^{1}$ Ara Institute of Canterbury, Christchurch, Aotearoa New Zealand ${ }^{2}$ Corresponding Author: trish.thomson@ara.ac.nz

\section{Citation}

Thomson, P., Richardson, A., \& Foster, G. (2021). Collaborative learning in the COVID19 pandemic: A change to the delivery of undergraduate nursing education. Nursing Praxis in Aotearoa New Zealand, 37(3), 34-36. https://doi.org.10.36951/27034542.2021.032

\begin{abstract}
At the time of the first lockdown in Aotearoa New Zealand, Bachelor of Nursing students were deemed non-essential workers and unable to complete clinical placements. This reflective piece describes an innovative solution to design meaningful learning activities for clinical placements in primary health care settings. With a focus on collaborative learning in a virtual team, student nurses participated in a project which focused on disaster nursing preparedness and management of the sequelae associated with a disaster, particularly the COVID-19 pandemic. Appropriate e-learning short courses contributed to student preparation for clinical practice acting as a substitute to clinical experience. The learning outcomes for student nurses included enhanced teamwork, demonstration of leadership, relevant learning that enabled completion of the Bachelor of Nursing programme with work readiness.
\end{abstract}

Keywords: COVID-19; e-learning; online learning; undergraduate nursing education

\section{Introduction}

The emergence of the COVID-19 global health pandemic saw public health and social measures disrupt and restrict student nurse learning. Clinical placements were not feasible in primary health care settings due to the changed nature of practice and the uncertainty of the progression of the pandemic. Using the self-reflective framework provided by Rolfe, Freshwater and Jasper (2001) of 'what? so what? and now what?' an innovative solution, which created a change to the delivery of undergraduate nursing education, is explored.

\section{What?}

Last year in 2020, Aotearoa New Zealand introduced a four-level alert system with the onset of the COVID19 pandemic in January 2020 (Ministry of Health, [MOH], 2020a). By March 2020, a state of emergency and alert level four lockdown had been applied $\mathrm{MOH}$, 2020a). At that time, the Chief Nursing Officer advised nurse leaders that student safety was paramount and that nursing students should not attend clinical placement as they were deemed non- essential workers (MOH, 2020b). In response, Bachelor of Nursing (BN) academic staff were compelled to pivot and rethink delivery of the year three clinical course 'Family, Whānau, and Community nursing' (Ara Institute of Canterbury, 2021). Within this new environment, effective preceptorship, registered nurse oversight, alignment to learning objectives, and competency assessments in clinical settings and environments were not possible. Prioritising year three nursing students' learning and progression in the $\mathrm{BN}$ programme was necessary to ensure their entry into the registered nurse workforce in 2021. Consequently, a learning experience that could mirror an authentic primary health care and community nursing clinical experience was designed and implemented with the Nursing Council of New Zealand approval.

\section{So what?}

Teamwork within the experienced academic staff team was imperative to design and develop an authentic and relevant clinically based disaster preparedness project for nursing students. Prior 
research informed the content in supporting flexibility of course delivery, acknowledging the sequelae of crisis events, and informing the actual and potential roles of nursing in disaster events (Richardson et al., 2015). The project included a focus on collaborative learning within a team and provided an opportunity for nursing students to recognise their individual strengths and challenges within their own current skill set (Barton et al., 2018). Learning to work together within a virtual team also highlighted various interpersonal dynamics and nursing students observed and reflected on how their behaviours influenced their own individual performance, including leadership roles and responsibilities (Logan, 2016). Students participated in learning and teaching activities through self-reflection, virtual and digital platforms. Disaster nursing preparedness, and management of the sequelae associated with a disaster, were included in the project, with a focus on the COVID-19 pandemic. Appropriate e-learning short courses were included such as the use of personal protective equipment, safe vaccine storage and transportation, and provisional vaccinator foundation knowledge to prepare the students to return to the clinical setting. The project was supported by the clinical teaching team, including the authors.

\section{Now what?}

Upon reflection, it was recognised that the academic team's motivation, deep understanding of nursing pedagogy, experience as registered nurses, along with positivity and teamwork were key drivers to enable timely continuation of nursing students' learning within this course. The outcomes of this learning for the students included enhanced teamwork, demonstration of leadership, relevant and applicable knowledge with an emphasis on work readiness as future registered nurses. The implication for nursing education is that an appropriate learning experience can be substituted for a clinical learning experience in exceptional times and circumstances. Further outcomes have arisen through this project, which have included enhanced clinical skills, namely venepuncture, vaccination, and the unique opportunity for nursing students to gain clinical experiences at Managed Isolation Quarantine Facilities (MIQF). The MIQF multi-disciplinary team of police, military, nursing, and medical staff established a safe and supportive learning environment for nursing students through procedures and protocols. Indeed, the MIQF experience has led to employment for current graduates of the BN programme. "As we have seen throughout history, nurses are well able to think outside the box, and develop creative and innovative solutions to all manner of problems, conundrums and challenges" (Jackson et al., 2020, p. 2041).

\section{Concluding thoughts}

Nursing student project teams demonstrated innovation, realising the need for self-care in professional practice, enabling self-confidence in infection control principles, health education and the value of lifelong learning. Research is underway to capture the learning of nursing students and adaptability to change, including the experience of clinical placement in MIQF. Ongoing curriculum development will focus on innovative approaches to teaching and learning incorporating currency to nursing practice, disaster responsiveness, and workforce ready graduates.

\section{References}

Ara Institute of Canterbury. (2021). Family Whānau and Community Nursing.

https://www.ara.ac.nz/products/formal/7/bnfw700family-whanau-and-community-nursing/

Barton, G., Bruce, A., \& Schreiber, R. (2018). Teaching nurses' teamwork: Integrative review of competencybased team training in nursing education. Nurse Education in Practice, 32, 129-137. https://doi.org/10.1016/j.nepr.2017.11.019

Jackson, D., Bradbury-Jones, C., Baptiste, D., Gelling, L., Morin, K., Neville, S., \& Smith, G. D. (2020). Life in the pandemic: Some reflections on nursing in the context of COVID-19. Journal of Clinical Nursing, 29(13-14), 2041-2043. https://doi.org/10.1111/jocn.15257

Logan, T. R. (2016) Influence of teamwork behaviors on workplace incivility as it applies to nurses. Creighton Journal of Interdisciplinary Leadership, 2(1), 47-53. https://dx.doi.org/10.17062/CJIL.v2il.28

Ministry of Health. (2020a, March 26). Chief Nursing Officer letter to nurse leaders.

https://www.health.govt.nz/system/files/documents Lpages/cno-letter-nurse-leaders-covid1926mar20.pdf

Ministry of Health. (2020b, December 15). About the Alert System. https://covid19.govt.nz/alert-system/aboutthe-alert-system

Richardson, S. K., Richardson, A., Trip, H., Tabakakis, K., Josland, H., Maskill, V., Dolan, B., Hickmott, B., Houston, G., Cowan, L., \& McKay, L. (2015). The impact of a natural disaster: Under- and post graduate nursing education following the Canterbury, New Zealand, 
earthquake experiences. Higher Education Research and Development, 34, 986-1000

https://dx.doi.org/10.1080/07294360.2015.1011099

Rolfe, G., Freshwater, D., \& Jasper, M. (2001). Critical reflection for nursing and the helping professions: $A$ user's guide. Palgrave MacMillan Ltd.

\section{Funding: None}

\section{Conflict of interest: None}

